\title{
ChemComm
}

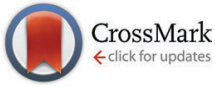

Cite this: Chem. Commun., 2015, 51, 12369

Received 24th March 2015,

Accepted 12th June 2015

DOI: $10.1039 / c 5 c c 02429 c$

www.rsc.org/chemcomm

\section{Plasmonic Ge-doped ZnO nanocrystals $\dagger$}

\author{
Enrico Della Gaspera, ${ }^{* a}$ Noel W. Duffy, ${ }^{b}$ Joel van Embden, ${ }^{a}$ Lynne Waddington, ${ }^{a}$ \\ Laure Bourgeois, ${ }^{C}$ Jacek J. Jasieniak $\ddagger^{*^{a}}$ and Anthony S. R. Chesman ${ }^{{ }^{a}}$
}

\begin{abstract}
We present the first colloidal synthesis of Ge-doped $\mathrm{ZnO}$ nanocrystals, which are produced by a scalable method that uses only air and moisture stable precursors. The incorporation of tetravalent $\mathrm{Ge}$ ions within $\mathrm{ZnO}$ nanocrystals generates a surface plasmon resonance in the near-mid infrared, and induces a change in morphology, from isotropic spheroidal nanocrystals to rod-like, elongated structures with a distinctive $c$-axis orientation.
\end{abstract}

The ability to precisely incorporate dopants into colloidal $\mathrm{ZnO}$ nanocrystals (NCs) has created a reliable pathway to tailoring the optical and electronic properties of $\mathrm{ZnO}$. As a consequence, doped $\mathrm{ZnO}$ has application in electrochemistry, photocatalysis, optoelectronics, magnetism and sensing. ${ }^{1-5}$ In particular, doped ZnO NCs displaying a localized surface plasmon resonance (LSPR) have recently become an attractive alternative to expensive indium tin oxide (ITO). ${ }^{6-10}$ Plasmonic ZnO NCs have typically been doped with trivalent cations via substitution at the zinc site, with the dopant providing free charge carriers that give rise to a plasmonic resonance centered in the near or mid infrared. Due to the similarity in valence and cation size between trivalent substituents (typically $\mathrm{Al}$, Ga or In) and zinc ions, such dopants can be incorporated within $\mathrm{ZnO}$ at relatively high concentrations. ${ }^{5-7,11}$ In contrast, investigations into doping $\mathrm{ZnO}$ with tetravalent p-block elements by solution-based routes have been limited,${ }^{12}$ presumably owing to the challenges

\footnotetext{
${ }^{a}$ CSIRO Manufacturing Flagship Ian Wark Laboratories, Bayview Ave, Clayton, Victoria 3168, Australia. E-mail: enrico.dellagaspera@csiro.au,

Jacek.Jasieniak@monash.edu, anthony.chesman@csiro.au

${ }^{b}$ CSIRO Energy Flagship, Ian Wark Laboratories, Bayview Ave, Clayton, Victoria 3168, Australia

${ }^{c}$ Monash Centre for Electron Microscopy and Department of Materials Science and Engineering, Monash University, Wellington Road, Clayton, Victoria 3800, Australia

$\dagger$ Electronic supplementary information (ESI) available: Experimental details, FTIR spectra, additional UV-Vis-NIR, XRD and TEM with size distributions and EDS data, SEM of thin films, full characterization of $\mathrm{ZnO}: \mathrm{Si}$ and $\mathrm{ZnO}: \mathrm{Sn}$ nanocrystals. See DOI: $10.1039 / \mathrm{c} 5 \mathrm{cc} 02429 \mathrm{c}$

\# Present address: Materials Science and Engineering, Monash University, Wellington Rd, Clayton, Victoria 3800, Australia.
}

associated with the disparity in the physical and electronic properties between the dopant and host cations.

In light of this, germanium is the most suitable candidate among the Group IV elements to act as a 4+ dopant in $\mathrm{ZnO}$, as it is closer in size to $\mathrm{Zn}$ than $\mathrm{Si}$, and has a more stable tetravalent oxidation state than $\mathrm{Sn}$. Ge-doped $\mathrm{ZnO}$ (ZnO:Ge) has been examined for its potential use as a photoluminescent material and a transparent conducting oxide (TCO). To date, its synthesis has been limited to the use of expensive vacuum-based deposition techniques, such as sputtering, ${ }^{13}$ evaporation,,${ }^{14}$ and ALD,${ }^{15}$ or high temperature liquid phase epitaxy, ${ }^{16}$ with no solution-based methods amenable to cheap, large scale synthesis available.

In this work, we overcome this barrier by presenting the first synthesis of Ge-doped ZnO colloidal NCs. The synthetic method developed here utilizes a scalable heat-up technique instead of the commonly adopted hot-injection protocol. ${ }^{17}$ It employs only air and moisture stable precursors, and provides ready access to large quantities of ZnO:Ge NCs across a range of doping levels. We examine in detail the effect of the dopant concentration on the optical properties and the morphology of the NCs. Furthermore, we demonstrate the general applicability of this method by synthesizing ZnO NCs doped with Si and Sn.

ZnO:Ge NCs were synthesized by an adapted colloidal heat-up method previously developed for the formation of $\mathrm{Al}, \mathrm{Ga}$, and In-doped ZnO NCs. ${ }^{11}$ Briefly, an air stable Ge precursor solution was first formed by heating $\left[\mathrm{Ge}\left(\mathrm{O}, \mathrm{O}^{\prime} \text {-glycolate }\right)_{2}\left(\mathrm{H}_{2} \mathrm{O}\right)_{2}\right]$ in oleylamine at $120{ }^{\circ} \mathrm{C}$ under vacuum. ${ }^{18}$ Upon cooling, the Ge precursor was added to zinc stearate suspended in oleic acid, 1-dodecanol and octadecene. The solution was then heated at $230{ }^{\circ} \mathrm{C}$ for 2 hours to give ZnO:Ge NCs (see ESI $\dagger$ for experimental procedures). The samples have been labeled $\mathrm{ZGe} X$ where $X$ is the nominal Ge/Zn ratio in atomic \%. The reaction proceeds via esterification between the carboxylate groups coordinating the metals and the hydroxyl moieties of the alcohol, which results in the formation of metal hydroxide species that ultimately condense to give doped $\mathrm{ZnO}$ NCs. ${ }^{11}$

Optical characterization of the as-prepared colloids is presented in Fig. 1. A distinctive IR feature was observed in the 

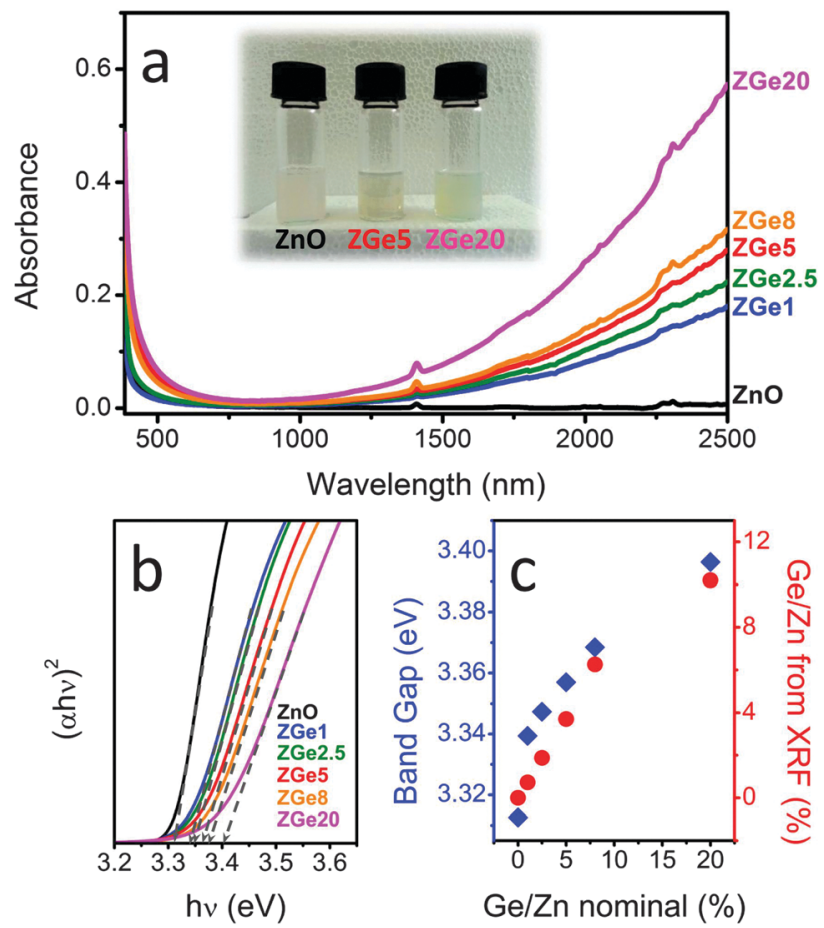

Fig. 1 (a) Optical absorption spectra of equimolar colloidal solutions of $\mathrm{ZnO}: \mathrm{Ge}$ in tetrachloroethylene (TCE). The inset shows a digital picture of the prepared colloidal solutions. (b) Tauc plot (exponent $=2$, direct band gap) of the $\mathrm{ZnO}: \mathrm{Ge}$ colloidal solutions. The dashed lines are the linear fits used to identify the band gap. (c) Optical band gap and real Ge/Zn amount evaluated from XRF as a function of the nominal Ge/Zn ratio.

optical absorption spectra of all Ge-doped NC colloidal solutions (Fig. 1a and Fig. S1, ESI $\dagger$ ). Examination of Fig. 1a reveals that the magnitude of the absorption in this region increased concomitantly with Ge doping levels. We ascribe this feature to a LSPR peak arising from dopant-induced generation of free electrons. ${ }^{6,11,19}$ Consequently, the doped solutions have a pale green color, in comparison to the colorless undoped $\mathrm{ZnO}$ (inset of Fig. 1a). This distinctive coloring has also been observed previously for $\mathrm{Al}-$, Ga- and In-doped $\mathrm{ZnO} \mathrm{NCs}$, and it is ascribed to the presence of aliovalent dopants. ${ }^{5,11}$ Concurrent with higher Ge doping levels, the optical band gap of $\mathrm{ZnO}$ increased as predicted by the Burstein-Moss effect (Fig. 1b and c). Fig. 1c shows that as the nominal Ge doping level is raised from 0 to $20 \%$ the band gap increases from $\sim 3.31 \mathrm{eV}$ to $3.4 \mathrm{eV}$.

Confirmation of the extent of doping was achieved by X-ray fluorescence (XRF) analysis, which was used to evaluate the real Ge/Zn ratio (Fig. 1c and Table S1, ESI $\dagger$ ). The doping efficiency, evaluated as the ratio of Ge incorporated within the NCs with respect to the amount of precursor used, was approximately $75 \%$ for all Ge precursor concentrations examined in this study, excluding the highest, which gave a doping efficiency of $\sim 50 \%$. The decrease in doping efficiency at higher dopant precursor loadings has been observed previously. ${ }^{11,20}$

X-ray diffraction (XRD) analyses confirmed that all of the prepared materials were in the wurtzite crystalline phase (ICDD No. 34-1451) with no secondary or contaminating phases (e.g., $\mathrm{GeO}_{2}, \mathrm{ZnGeO}_{3}, \mathrm{Zn}_{2} \mathrm{GeO}_{4}$ ) observed (Fig. 2a). A slight shift in

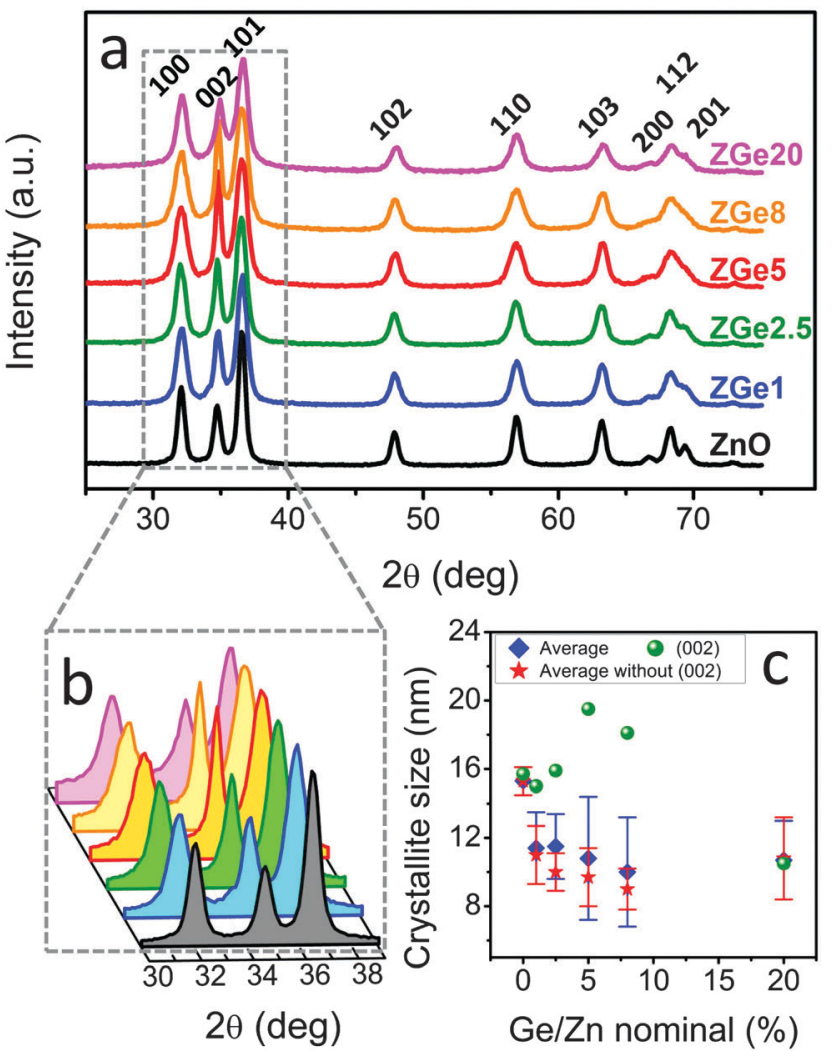

Fig. 2 (a) XRD patterns of $\mathrm{ZnO}: G e$ powders. The diffraction patterns are offset vertically for clarity and the peaks are indexed according to the ICDD No. 34-1451 reference. (b) Zoomed view of the (100), (002) and (101) diffraction peaks. (c) Crystallite size of the prepared NCs as a function of $\mathrm{Ge}$ doping. The error bars are plus or minus one standard deviation. By excluding the (002) diffraction peak in the estimation of the crystallite size, smaller sizes with lower standard deviations are observed.

diffraction peaks towards higher angles was observed with increasing Ge doping levels, consistent with a reduction in the crystal lattice parameters following the substitution of $\mathrm{Zn}^{2+}$ with smaller $\mathrm{Ge}^{4+}$ ions (Fig. S2, ESI $\dagger$ ). ${ }^{15}$ The broadening of the diffraction peaks is consistent with the crystalline domains being in the nanometer size regime (Fig. S3, ESI $\dagger$ ). The crystallite size evaluated with the Scherrer relationship was $\sim 15 \mathrm{~nm}$ for the undoped NCs, and slightly smaller (10-12 nm) for the doped NCs, regardless of the level of doping. This is possibly due to the stressinduced reduction in crystal growth caused by the incorporation of dopants within the $\mathrm{ZnO}$ lattice. ${ }^{6,21}$ In addition to the dopant-induced decrease in crystallite size, a progressive increase in intensity and reduction in width of the (002) diffraction peak with increasing Ge doping levels was observed (Fig. $2 b$ and c). This phenomenon is usually ascribed to the formation of elongated structures oriented along the $c$-axis of the hexagonal wurtzite cell, which is the most energetically favourable growth direction. ${ }^{22,23}$ This is in agreement with the morphology of the as-prepared ZnO:Ge NCs synthesised here (vide infra).

Collectively, these analyses confirmed the successful incorporation of Ge ions within the ZnO NCs, proving the efficacy of our optimized synthetic protocol. While Ge dopants were responsible for the LSPR in the infrared spectrum, their presence inside the 

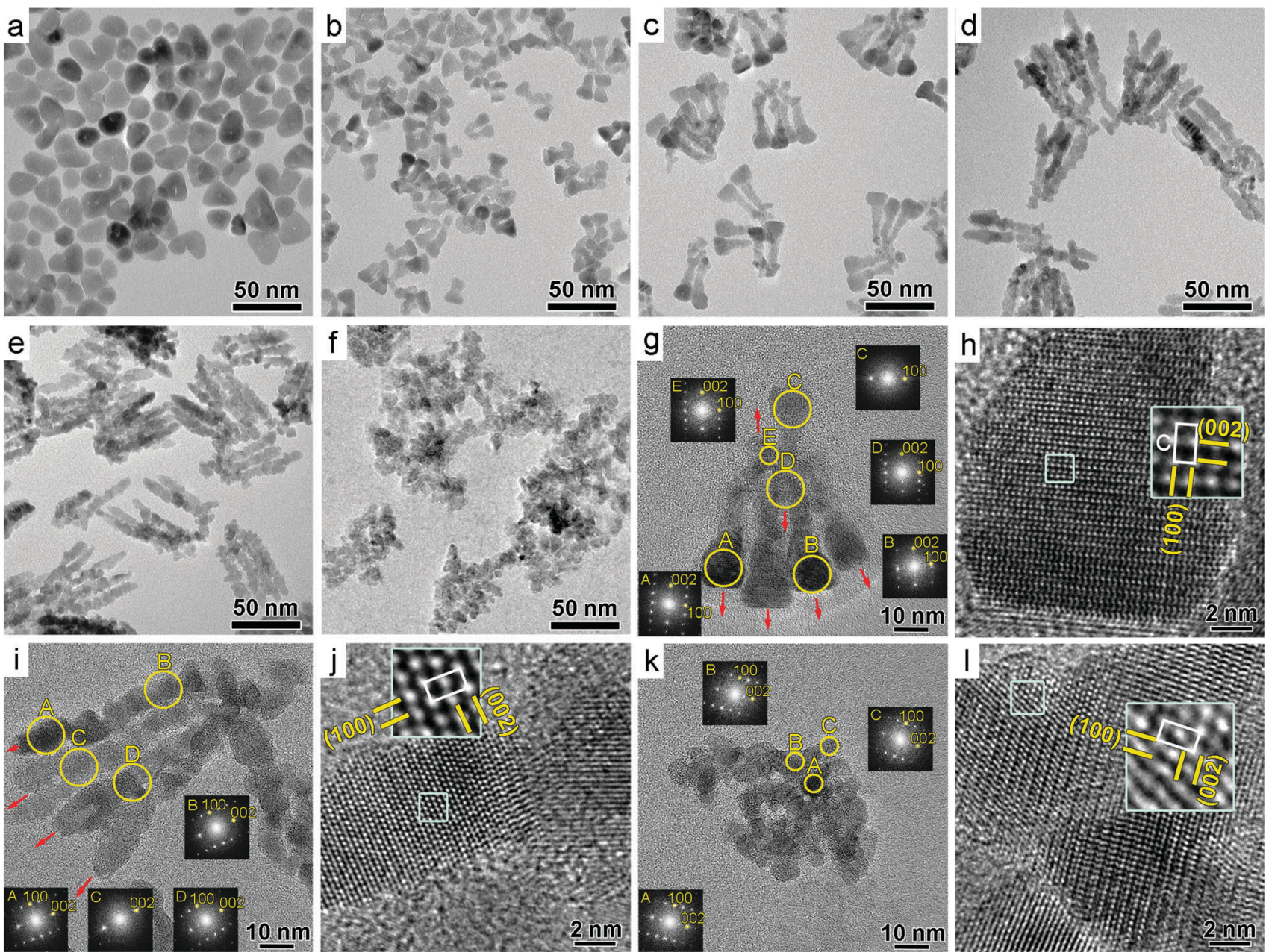

Fig. 3 (a-f) Low-resolution TEM images of (a) ZnO, (b) ZGe1, (c) ZGe2.5, (d) ZGe5, (e) ZGe8 and (f) ZGe20 NCs. (g-l) High resolution TEM images of (g, h) ZGe2.5, (i, j) ZGe5 and (k, l) ZGe20. The red arrows highlight the $c$-axis direction. Fast Fourier transforms obtained from the circular regions in $\mathrm{g}$, $\mathrm{i}$ and $\mathrm{k}$ indicated good crystallinity and preferred growth along [001] for ZGe2.5 and ZGe5. The insets in $\mathrm{h}, \mathrm{j}$ and I show enlarged views of the boxed area together with the unit cell of the $\mathrm{ZnO}$ crystal structure, where $c$ is the $c$ lattice parameter $(c=5.20 \AA) .{ }^{27}$

ZnO lattice also affected the size and the morphology of the NCs. Without any dopants, isotropic, almost spherical ZnO NCs, with diameters in the $15-20 \mathrm{~nm}$ range, were formed (Fig. 3a and Fig. S4, ESI $\dagger$ ). The introduction of only $1 \%$ of the Ge precursor into the reaction solution caused the formation of substantially smaller, slightly elongated particles, composed of a pod extruding from a slightly bigger center (Fig. 3b and Fig. S5, ESI†). This transformation became more pronounced when additional Ge was incorporated (Fig. 3c and Fig. S6, ESI †).

At 5\% nominal doping, faceted, slightly irregular nanorods that do not display a distinct center were formed (Fig. 3d and Fig. S7, ESI $\dagger$ ). Concurrent with this morphological transformation, a reduction in the lateral size (width) and an increase in the length of the NCs with respect to the undoped $\mathrm{ZnO}$ particles occurred. Such nanorods appeared to be composed of small NCs merged together, a phenomenon that has been previously observed in chain-like rods of $\mathrm{ZnO}^{24}$ or other semiconducting NCs. ${ }^{25,26}$

High-resolution transmission electron microscopy (HRTEM) images showed that ZGe2.5 and ZGe5 contained highly crystalline particles that are strongly aligned along the $c$-axis (red arrows in
Fig. $3 g$ and i), which explains the narrower and more intense (002) diffraction peaks at higher doping levels. ZGe8 NCs had a very similar morphology to ZGe5 NCs, although the rods are somewhat shorter on average (Fig. 3e and Fig. S8, ESI†).

Interestingly, the very high Ge doping levels achieved in ZGe20 caused the NCs to lose their distinctive elongated morphology and become clusters of randomly merged NCs (Fig. $3 \mathrm{f}$ and Fig. S9, ESI $\dagger$ ). The stress on the ZnO lattice induced by the excessive level of doping may have limited preferential growth along the $c$-axis of the hexagonal wurtzite cell. This may also be the cause of the shorter rods observed in ZGe8 in comparison to ZGe5. To support this hypothesis, the lattice spacings were measured from the Fourier transforms of the HRTEM images. The lattice parameter along the $[00 l]$ direction was found to be significantly reduced in ZGe2.5 and ZGe5 NCs (typically by $2 \%$, well above the measurement uncertainty of $0.7 \%$ ), while the other directions were hardly affected (Fig. S10, ESI $\dagger$ ). Interestingly, ZGe20 NCs have a wider spread of lattice parameters in all directions, originating from their highly disordered structure, indicating some degradation of the 
crystal lattice due to excessive doping. From HRTEM it can be concluded that lattice shrinkage started along the $[00 l]$ direction, which corresponds to the family of lattice planes with the highest surface energy, ${ }^{28}$ and then spread to the other [uv0] directions as doping increases, eventually compromising the structural integrity of the NCs. The preferential shrinkage along the $c$-axis at low doping levels has been previously observed in Al-doped ZnO NCs, and it has been ascribed to a possible preferential site occupation by $\mathrm{Al}^{3+}$ ions. $^{6}$

The evolution of the NCs as a function of the reaction time was investigated using optical absorption spectroscopy and TEM; Ge dopants were found to be continuously incorporated within the $\mathrm{ZnO}$ NCs over the duration of the $2 \mathrm{~h}$ reaction, with the rate of incorporation decreasing with time (Fig. S12, ESI $\dagger$ ). Such a decrease is more evident when the initial amount of Ge precursor was low (ZGe5), because the dopant source is rapidly depleted from the solution during the initial stages of the reaction. Conversely, at high Ge loadings (ZGe20), there was sufficient Ge precursor available to promote a higher rate of doping during the later stages of the $2 \mathrm{~h}$ reaction. The heavily doped $\mathrm{ZnO}$ NCs required more time to nucleate, with no ZGe20 NCs isolatable 5 minutes after the reaction solution reached $230{ }^{\circ} \mathrm{C}$, while ZGe5 NCs were already fully formed in that period of time, which is additional evidence that the Ge precursor plays a key role in the nucleation, growth and consequent morphology of the Ge-doped ZnO NCs. Interestingly, the NCs do not change morphology during the course of the reaction (see Fig. S13 and S14, ESI $\dagger$ ), while as discussed before, the doping is found to constantly increase over time.

As a proof-of-concept study, the versatile reaction method presented here was also applied to the synthesis of ZnO NCs doped with other p-block elements, namely Si and Sn (Fig. S15S18 and Table S1, see ESI $\dagger$ for details), confirming its wider applicability towards the preparation of a variety of doped NCs.

Ge-doped ZnO NCs can be dispersed in various organic solvents (e.g. octane, toluene) at concentrations of up to $200 \mathrm{mg} \mathrm{mL} \mathrm{m}^{-1}$. These could be used to prepare high quality thin films through several deposition methods, such as spin coating, spray coating, drop casting, and printing (see Fig. S19 for SEM and optical characterization of spin coated films, ESI $\dagger$ ). The ability to fabricate functional thin films from NC inks makes doped $\mathrm{ZnO}$ colloids attractive for a variety of applications in optoelectronics, sensing and catalysis.

In conclusion, we have developed the first synthesis of Ge-doped ZnO colloidal NCs with plasmonic resonances in the infrared spectrum. The synthesis is based on a scalable, heat-up protocol which utilizes only air and moisture stable reagents. Ge dopants were found to strongly affect not just the functional properties of the $\mathrm{ZnO} \mathrm{NCs}$, but also their morphology, with a progressive transformation from isotropic nanoparticles to elongated nanorods, and eventually at very high concentrations $(>10 \%$ atomic) to clusters of merged NCs. This work constitutes a significant step forward towards further investigations on $\mathrm{ZnO}$ doped with Group IV elements.

This work was funded through the Manufacturing Flagship of CSIRO as part of Office of the Chief Executive Postdoctoral
Fellowships (E.D.G., J.v.E.). J.J.J. and A.S.R.C. acknowledge the Australian Research Council for funding through Grant DP110105341 and for a Discovery Early Career Research Award, respectively. Steve Peacock and Thomas R. Gengenbach are thanked for XRF and XPS measurements, respectively. The authors acknowledge use of facilities within the Monash Centre for Electron Microscopy and the support of the State of Victoria and Monash University for instrumentation.

\section{Notes and references}

1 P. V. Radovanovic, N. S. Norberg, K. E. McNally and D. R. Gamelin, J. Am. Chem. Soc., 2002, 124, 15192-15193.

2 V. Etacheri, R. Roshan and V. Kumar, ACS Appl. Mater. Interfaces, 2012, 4, 2717-2725.

3 E. Della Gaspera, M. Guglielmi, G. Perotto, S. Agnoli, G. Granozzi, M. L. Post and A. Martucci, Sens. Actuators, B, 2012, 161, 675-683.

4 G. Garcia, R. Buonsanti, A. Llordes, E. L. Runnerstrom, A. Bergerud and D. J. Milliron, Adv. Opt. Mater., 2013, 1, 215-220.

5 J. Song, S. A. Kulinich, J. Li, Y. Liu and H. Zeng, Angew. Chem., Int. Ed., 2015, 54, 462-466.

6 R. Buonsanti, A. Llordes, S. Aloni, B. A. Helms and D. J. Milliron, Nano Lett., 2011, 11, 4706-4710.

7 S. Ghosh, M. Saha and S. K. De, Nanoscale, 2014, 6, 7039-7051.

8 X. Liang, Y. Ren, S. Bai, N. Zhang, X. Dai, X. Wang, H. He, C. Jin, Z. Ye, Q. Chen, L. Chen, J. Wang and Y. Jin, Chem. Mater., 2014, 26, 5169-5178.

9 E. Della Gaspera, M. Bersani, M. Cittadini, M. Guglielmi, D. Pagani, R. Noriega, S. Mehra, A. Salleo and A. Martucci, J. Am. Chem. Soc., 2013, 135, 3439-3448.

10 S. Cimitan, S. Albonetti, L. Forni, F. Peri and D. Lazzari, J. Colloid Interface Sci., 2009, 329, 73-80.

11 E. Della Gaspera, A. S. R. Chesman, J. van Embden and J. J. Jasieniak, ACS Nano, 2014, 8, 9154-9163.

12 S. Ghosh, M. Saha, V. Dev Ashok, B. Dalal and S. K. De, J. Phys. Chem. C, 2015, 119, 1180-1187.

13 D. H. Fan, Z. Y. Ning and M. F. Jiang, Appl. Surf. Sci., 2005, 245, 414-419.

14 Y. Su, X. Meng, Y. Chen, S. Li, Q. Zhou, X. Liang and Y. Feng, Mater. Res. Bull., 2008, 43, 1865-1871.

15 P. R. Chalker, P. A. Marshall, P. J. King, K. Dawson, S. Romani, P. A. Williams, J. Ridealgh and M. J. Rosseinsky, J. Mater. Chem., 2012, 22, 12824-12829.

16 D. Ehrentraut, H. Sato, Y. Kagamitani, A. Yoshikawa, T. Fukuda, J. Pejchal, K. Polak, M. Nikl, H. Odaka, K. Hatanaka and H. Fukumura, J. Mater. Chem., 2006, 16, 3369-3374.

17 J. Van Embden, A. S. R. Chesman and J. Jasieniak, Chem. Mater., 2015, 27, 2246-2285.

18 A. S. R. Chesman, J. van Embden, E. Della Gaspera, N. W. Duffy, N. A. S. Webster and J. J. Jasieniak, Chem. Mater., 2014, 26, 5482-5491.

19 A. Comin and L. Manna, Chem. Soc. Rev., 2014, 43, 3957-3975.

20 L. De Trizio, R. Buonsanti, A. M. Schimpf, A. Llordes, D. R. Gamelin, R. Simonutti and D. J. Milliron, Chem. Mater., 2013, 25, 3383-3390.

21 Z. Lu, J. Zhou, A. Wang, N. Wang and X. Yang, J. Mater. Chem., 2011, 21, 4161-4167.

22 Y. Yang, Y. Jin, H. He, Q. Wang, Y. Tu, H. Lu and Z. Ye, J. Am. Chem. Soc., 2010, 132, 13381-13394.

23 E. Della Gaspera, J. van Embden, A. S. R. Chesman, N. W. Duffy and J. J. Jasieniak, ACS Appl. Mater. Interfaces, 2014, 6, 22519-22526.

24 C. Pacholski, A. Kornowski and H. Weller, Angew. Chem., Int. Ed., 2002, 41, 1188-1191.

25 K.-S. Cho, D. V. Talapin, W. Gaschler and C. B. Murray, J. Am. Chem. Soc., 2005, 127, 7140-7147.

26 Z. Tang, N. A. Kotov and M. Giersig, Science, 2002, 297, 237-240.

27 Attempts to map the distribution of the Ge-dopant in the ZnO lattice by X-ray energy dispersive spectroscopy (XEDS) was not successful, as although Ge is clearly detected, it cannot be mapped with confidence due to the small amount of Ge, overlap between Ge and $\mathrm{Zn}$ signals, and beam damage to the NCs after prolonged electron beam exposure (Fig. S11, ESI $\dagger$ ).

28 C. Wöll, Prog. Surf. Sci., 2007, 82, 55-120. 